(C)2009 IEEE. Personal use of this material is permitted. However, permission to reprint/republish this material for advertising or promotional purposes or for creating new collective works for resale or redistribution to servers or lists, or to reuse any copyrighted component of this work in other works must be obtained from the IEEE. 


\title{
An improved approach to estimate soft tissue parameters using genetic algorithm for minimally invasive measurement
}

\author{
Yashar Madjidi*a, Bijan Shirinzadeh ${ }^{\mathrm{a}}$, Reza Banirazi ${ }^{\mathrm{a}}$, Yanling Tian ${ }^{\mathrm{a}}$, Julian Smith ${ }^{\mathrm{b}}$, \\ Yongmin Zhong ${ }^{\mathrm{c}}$ \\ ${ }^{a}$ Robotics \& Mechatronics Research Laboratory, Department of Mechanical \& Aerospace Engineering, \\ Monash University, Clayton, Victoria 3800, Australia \\ ${ }^{\mathrm{b}}$ Department of Surgery, Monash Medical Centre, Monash University, Clayton, Victoria 3800, Australia \\ ${ }^{\mathrm{c}}$ Department of Mechanical Engineering, Curtin University of Technology, WA 6845, Australia
}

\begin{abstract}
This paper evaluates the ability of a gradient-free estimation method using genetic algorithm (GA) to model the elastic stress response of the anterior cruciate ligament (ACL) based on quasi-linear viscoelastic $(Q L V)$ theory. The improved GA simultaneously fits the ramping and relaxation experimental data to the $Q L V$ constitutive equation to obtain the soft tissue parameters. This approach is then compared with a previously evaluated method for two exponential and polynomial QLV models. The earlier approaches are mainly based on regression algorithms, which usually try to find a gradient-based solution with probability of poor convergence and variability of constants. Contrarily, this paper presents a gradient-free algorithm based on the improved timesaving GA. The results demonstrate that the ability of this algorithm to estimate the QLV parameters in timesaving process is functional to develop the optimal methodology for minimally invasive measurement during surgery.
\end{abstract}

\section{Introduction}

A real time and realistic organ-force modeling of physics-based behavior of soft tissue is a promising step to define operational limitations for robotic endeffectors palpating the biological soft tissue. However such process is extremely challenging not only because of the non-linearity, rate, and time dependence of an organ's material properties but also because of organ tissues' layered and non-homogeneous structure [1-5]. In order to intra-operative characterization of biological tissues through in-vitro experiments by robotic indenters, considerable methods have been introduced mainly by using explicit FEM solutions [2]. One of the main goals of such researches aims at establishing the accurate and time-saving computational process.

In attempting to make the optimum intra-operative characterization, this paper investigated the estimation ability of a direct search tool to calculate the parameters of a physics-based model.

${ }^{*}$ Corresponding author.

E-mail: Yashar.Madjidi@eng.monash.edu.au
While the QLV model has been widely used to characterize viscoelastic behavior, a few studies have focused on the ability of this model to estimate the overall stress-strain response of ligaments and tendons $[6,7]$. The goal of this study is to evaluate an improved GA approach to model the elastic stress response of the ACL based on QLV theory. The result is compared with an exponential formulation model [8] and a polynomial form of the Mooney-Rivlin (MR) model [9] with the instantaneous assumption approach. Moreover, as an improved GA method, the convergence of this algorithm has been investigated with two different crossover functions.

\section{Model and Algorithm}

\subsection{QLV model}

The most commonly discussed model in the biomechanics literature is the quasi-linear viscoelastic (QLV) model introduced by Fung (1981). Significantly, An improved method to analyze the stress relaxation of ligaments following a finite ramp time based on the QLV theory was introduced by Abramowitch and Woo to obtain the constants for QLV theory [10]. Through this method, the ramping and relaxation portions of the data simultaneously are fitted to the constitutive equation based on the strain history approach. The related equations are minimized using gradient based optimization algorithm. Recently, a direct search approach was applied based on a simple conventional GA to obtain QLV parameters [11].

According to the QLV theory, the complete stress in a tissue subjected to a step strain can be expressed by the following convolution formula

$$
\sigma(t)=G(t) * \sigma^{e}(\lambda)
$$

where $G(t)$ is the reduced relaxation function, $\sigma^{e}(\lambda)$ is the nonlinear elastic response, and $\lambda(t)$ is the stretch ratio. In general, $G(t)$ is a fourth-order tensor providing direction-dependent relaxation phenomena.

Using the Boltzmann superposition principle and representing the strain history as a series of 
infinitesimal step strains, the overall stress relaxation function can be expressed as the sum of all individual relaxations. For a general strain history, the stress at time $t$ is given by the convolution integral over time of $G(t)$ as follows

$$
\sigma(t)=\int_{-\infty}^{t} G(t-\tau) \cdot \frac{\partial \sigma^{e}(\lambda)}{\partial \lambda} \cdot \frac{\partial \lambda}{\partial \tau} \cdot d \tau
$$

where $\partial \sigma^{e}(\lambda) / \partial \lambda$ represents the instantaneous elastic response, and $\partial \lambda / \partial \tau$ is the stretch history. For biological soft tissues, it is commonly assumed that the relaxation function is the same and continuous in all directions which simplifies $G(t)$ to a scalar as

$$
G(t)=\frac{1+c \cdot\left[E_{1}\left(t / \tau_{1}\right)-E_{1}\left(t / \tau_{2}\right)\right]}{1+c \cdot \ln \left(\tau_{2} / \tau_{1}\right)}
$$

where $E_{1}(t)$ is the exponential integral function, and $c, \tau_{1}, \tau_{2}$ are the parameters determined from the experimental data. This relaxation function provides a smooth, linear decrease from short to long relaxation times. In some models, a decaying exponential equation has been chosen to describe the temporal behavior as the relaxation function [12]:

$$
G(t)=a e^{-b t}+c e^{-d t}+g e^{-h t}
$$

where coefficients $a, c$ and $g$, and exponents $b, d$ and $h$ are all constants to be determined experimentally.

Various constitutive laws can be considered to model elastic stress response of the soft tissue. In this study, the ability of an elastic model is evaluated through the integrated form of the QLV theory by utilizing GA approach and compared with MR model and a frequently-used exponential formulation.

2.1.1 Mooney-Rivlin (MR) model. The MR model is commonly used for estimation of soft tissue elastic stress responses [9]. In this model, the strain energy function may is expressed through Cauchy-Green tensor. The principal Cauchy stresses for an incompressible, isotropic material and in case of uniaxial tension is calculated by

$$
\sigma=2\left(\lambda^{2}-1 / \lambda\right) \cdot\left(C_{1}+C_{2} / \lambda\right) ; C_{1}+C_{2} \geq 0
$$

where $\sigma$ and $\lambda$ are the stress and stretch in the axial direction, respectively. The hydrostatic pressure term is determined from the equations of stress in the transverse directions. In order to maintain a positive strain energy function, the sum of the constants $C_{1}$ and $C_{2}$ in Eq. 5 must be greater than zero $[9,12]$. The axial stretch can be converted to the engineering strain using the following equation:

$$
\varepsilon=\frac{\Delta L}{L}=\lambda-1
$$

where $L$ is the length of the specimen and $\Delta L$ is the change of the length.

2.1.2 Exponential formulation. The exponential formulation has been widely used to describe the tensile behavior of ligaments and tendons. The empirical equation was firstly proposed in 1964 describing the tensile behavior of human skins as

$$
P=A\left(e^{B \varepsilon}-1\right) ; \quad A, B>0
$$

where $A$ and $B$ are material constants determined by fitting the model to the experimental data, and $\mathrm{P}$ and $\varepsilon$ represent engineering stress and strain. However, it does not consider 3D stress states and is not generally expressed in terms of a strain energy function [13]. Since other proposed exponential models were not successfully fitted to predict elastic stress-strain response for the tensile behavior of ligaments, those models are not taken into account in this study.

\subsection{Proposed genetic algorithm}

The GA can be applied to solve a variety of optimization problems that are specifically not well suited for standard optimization algorithms, including problems in which the objective function is discontinuous, non-differentiable, stochastic, or highly nonlinear [14]. The GA uses three main types of rules (selection, crossover, and mutation) at each step to create the next generation from the current population. This study has firstly focused on finding optimal conventional genetic operators and, as an improved method, briefly compared the convergence results through the proposed conventional crossover and realcoded one.

2.2.1 Fitness function. The population members are ranked on the basis of fitness function, and then typically their ranks are typically divided by the number of individuals to provide a probability threshold for selection.

In this study, the elastic model Eq. 5 and the relaxation function Eq. 4 are used in the QLV model Eq. 2. Considering engineering strain over the ramping period $\left(0<t<t_{0}\right)$, the stress resulted from a ramp test with a constant strain rate $(\gamma)$ can be written as

$$
\begin{aligned}
& \sigma\left(t: 0<t<t_{0}, \theta\right)= \\
& 2 \gamma \int_{0}^{t}\left\{a e^{-b(t-\tau)}+c e^{-d(t-\tau)}+g e^{-h(t-\tau)}\right\} . \\
& \left\{\left(C_{1}+\frac{C_{2}}{\gamma \tau+1}\right)\left(2(\gamma \tau+1)+\frac{1}{(\gamma \tau+1)^{2}}\right)-\right. \\
& \left.\frac{C_{2}\left((\gamma \tau+1)^{2}-1 /(\gamma \tau+1)\right)}{(\gamma \tau+1)^{2}}\right\} d \tau
\end{aligned}
$$

Similarly, the subsequent stress relaxation from $t_{0}$ to $t \rightarrow \infty$ can be described as 


$$
\begin{aligned}
& \sigma\left(t: t \geq t_{0}, \theta\right)= \\
& 2 \gamma \int_{0}^{t_{0}}\left\{a e^{-b(t-\tau)}+c e^{-d(t-\tau)}+g e^{-h(t-\tau)}\right\} . \\
& \left\{\left(C_{1}+\frac{C_{2}}{\gamma \tau+1}\right)\left(2(\gamma \tau+1)+\frac{1}{(\gamma \tau+1)^{2}}\right)-\right. \\
& \left.\frac{C_{2}\left((\gamma \tau+1)^{2}-1 /(\gamma \tau+1)\right)}{(\gamma \tau+1)^{2}}\right\} d \tau
\end{aligned}
$$

where $\theta=\left\{C_{1}, C_{2}, a, b, c, d, g, h\right\}$.

For a set of experimental data, the ramping portion of the data is defined as $\left(t_{i}, R_{i}\right)$ for $t: 0<t<t_{0}$ and the relaxation data as $\left(t_{i}, S_{i}\right)$ for $t_{0}<t<\infty$ [10]. Thus, the sum of square of the difference between the experimental data and the theory is described as

$$
\begin{aligned}
& f(\theta)=\sum_{i}\left[R_{i}-\sigma\left(t_{i}: 0<t_{i}<t_{0}, \theta\right)\right]^{2} \\
& g(\theta)=\sum_{i}\left[S_{i}-\sigma\left(t_{i}: 0<t_{i}<t_{0}, \theta\right)\right]^{2}
\end{aligned}
$$

The sum of the above functions of $\theta$ is considered as fitness function

$$
\text { Fitness Function }=f(\theta)+g(\theta)
$$

2.2.2 Population. The initialization of the population is usually done stochastically, though it is sometimes appropriate to start with one or more individuals that are selected heuristically. Regardless of the process used, the population should represent a wide assortment of individuals. The main trade-off on the size of populations is obvious, i.e. a large population will search the space more completely, but at a higher computational cost. It also seems that the sizes of populations tend to approximately increase with the individual string length linearly, rather than exponentially. However, the optimal population size (if one exists) depends on the problem as well. In this study, an initial size of population $n=200$ was firstly chosen and the best individual did have the highest fitness of all possibilities when population convergence occurred. To satisfy the time-saving requirement of minimally invasive measurement and surgery, the initial size of population $n=100$ was finalized according to the required precision for the fitness function.

2.2.3 Selection. The selection function chooses parents for the next generation based on their scaled values from the fitness scaling function. The main three widely used selection schemes are roulette wheel implementation, tournament selection, and elitism. The selection rule used in this study is based on tournament selection, while the size of tournament players for individuals is defined two. Tournament selection chooses each parent by considering the size of tournament players (individuals) at random and then choosing the best individual (the one with higher fitness) out of that set to be a parent.

2.2.4 Crossover. The most important operator in GA is crossover based on the metaphor of sexual combination. The two main attributes of crossover that can be varied are the probability of occurrences and the type of crossover implemented. The most basic crossover type is one-point crossover, which involves selecting a single crossover point at random and exchanging the portions of the individual strings to the right of the crossover point. Two-point crossover with a probability of $0.60-0.80$ is a relatively common choice as another type of crossover. The other type of crossover that has been found useful is called uniform crossover in which random decision is made at each bit position in the string as to whether or not to exchange (crossover) bits between the parent strings. Uniform crossover sometimes works better with slightly lower crossover probability. It is also common to start out running the GA with a relatively higher value for crossover, then taper off the value linearly to the end of the run, ending with a value of, say, one-half or twothirds the initial value [14]. In this study, considering the significant influence of crossover function and its fraction value on the convergence performance of GA, the behavior of this method was checked through different functions and fractions. As the result, single point function with less than 60 percent fraction value was chosen for the proposed conventional crossover. Since, with the conventional genetic operators, there is no guarantee the offspring are better than their parents. In this study, Direction-Based crossover (DBXO) has also been investigated. This operator uses the values of the objective function in determining the direction of genetic search. The operator generates single offspring $x^{\prime}$ from two parents $x_{1}$ and $x_{2}$ according to the following rule:

$x^{\prime}=r\left(x_{2}-x_{1}\right)+x_{2}$

where $\mathrm{r}$ is a random number between 0 and 1 . It also assumes that the parent $x_{2}$ is not worse than $x_{1}$; that is, $f\left(x_{2}\right) \leq f\left(x_{1}\right)$.

2.2.5 Mutation. Mutation is the stochastic flipping of bits occurred each generation. It is often done with a probability of something like 0.001 , but higher probabilities are not unusual. The probability of mutation is often held constant for the entire run of the GA, although this approach will not produce optimal results in many cases. It can be varied during the run, and if varied, it is usually increased. In this study, the fixed rate of 0.01 was defined for the mutation process and changing the mutation rate during the runs was not hypothesized as an influential operator.

New types of genetic operators or penalty functions are some of the differences between the improved and simple GA. The recently used improved GAs generally 
include new adaptive penalty schemes and adaptive mutations as well as adaptive crossover operators. In this study, the higher penalty factor than common values was found useful and especially setting higher value for the initial penalty after randomization of initial population was observed as a noticeable factor on the GA convergence performance.

\section{Performance Analysis}

Considering the availability of some estimated QLV models based on the same experimental data [12] and the importance of ACL as one of the most investigated tissues, this study has also used the same data to evaluate and predict the specified model through the proposed approach.

\subsection{Modeling of stress strain response}

Two elastic models (Eqs. 5 and 7) are used to fit the experimental data through a derivative-based method and a direct search solution. The experimental data is derived from previously published reports of uniaxial tensile tests of ACL $[7,12]$. The experimental data is estimated by digitizing images of the graphs through a raster to vector convertor software package. For derivative-based method, firstly each model is fitted to the stress-strain curve up to $4 \%$ strain based on a least squares fitting method using a mathematics software package (Mathematica, Wolfram Research). The elastic models are fitted assuming that the load is applied instantaneously. For derivative-free solution, GA as a direct-search tool is used to fit the stress-strain curve up to $4 \%$ strain using a technical computing software package (MATLAB, The MathWorks). All of the models are then used to model stress-strain behavior at higher levels of strain. The estimating ability of each model is evaluated as the models are fitted up to different levels of strain (Fig. 1). Exponential and MR formulations using derivativebased method are termed 'Exp.' and 'MR' respectively, and MR model using derivative-free method is termed as 'GA' on diagrams.

As shown in Fig. 1, below the 3\% strain level all the models closely fit the experimental data. Beyond this level, the ability of the models to fit the experimental curve is limited; however, the MR formulation (Eq. 5) models the stress response more accurately than the exponential formulation (Eq. 7). Considering the models to fit the experimental data up to $4 \%$ strain level (Fig. 1-b), at the $7 \%$ as for the final checking level with the experimental data, the exponential formulation overestimates the stress by $88 \%$ and the MR formulation does it by $15 \%$. Even by fitting the models to the experimental data up to $6 \%$ strain level (Fig. 1-c), at 7\% strain level, the exponential formulation overestimates the stress by $18 \%$ while the MR formulation overestimates by approximately $11 \%$.

The results of two elastic models through derivative-based method were previously reported [12]. Likewise, since the proposed derivative-free method in this study is used for MR model with the same precision of calculation, our method also indicated the same results. Generally speaking, the GA is more practical when finding a solution for multidimensional discrete problems is unavailable or time-consuming. Accordingly it should be noted that the proposed method is mainly followed to investigate the estimation of QLV parameters through stressrelaxation behavior.

\subsection{Modeling of stress relaxation response}

In derivation of the constitutive equation, as the first modeling step, choosing appropriate models for the instantaneous elastic response and the relaxation function is required. For tendons and ligaments, the exponential formulation based on Eq. 7 has been already used by some researchers [12]. For elastic and hyperelastic soft tissues, such as brain, the MR formulation and Ogden formulation has been widely used in the literature [12]. Also, the relaxation functions based on Eqs. 3 and 4 are widely used by assuming a continuous relaxation spectrum [13].

In this study, the elastic models (exponential and MR formulations) based on Eqs. 5 and 7 with Eq. 4 as the relaxation function are chosen to be used in the QLV model (Eq. 2) to model the stress relaxation response of the ACL. The related experimental data are derived from the previously reported and investigated data sets $[8,12,15]$. By using these two computing approaches, three different QLV models are investigated in this study. Two of QLV models comprise two elastic models and the exponential relaxation function Eq. 4. The parameters of these models are estimated through a derivative-based method by fitting the elastic model and the relaxation function separately, assuming that load is applied instantaneously. Although this method has been recommended and performed in the previous studies [12], the estimation of QLV parameters are investigated with the different relaxation functions in the present research. The third QLV model, as the main investigated QLV model in this study, comprises the MR elastic model and the exponential relaxation function using the GA as a derivative-free method to estimate QLV parameters. Similar to the previous studies $[10,11]$, the ramping and relaxation portions are simultaneously fitted to the constitutive equation in order to remove the assumption of a step change in strain. All the models are fitted to the stress relaxation data measured at the $2 \%$ strain level to determine the material constants. 


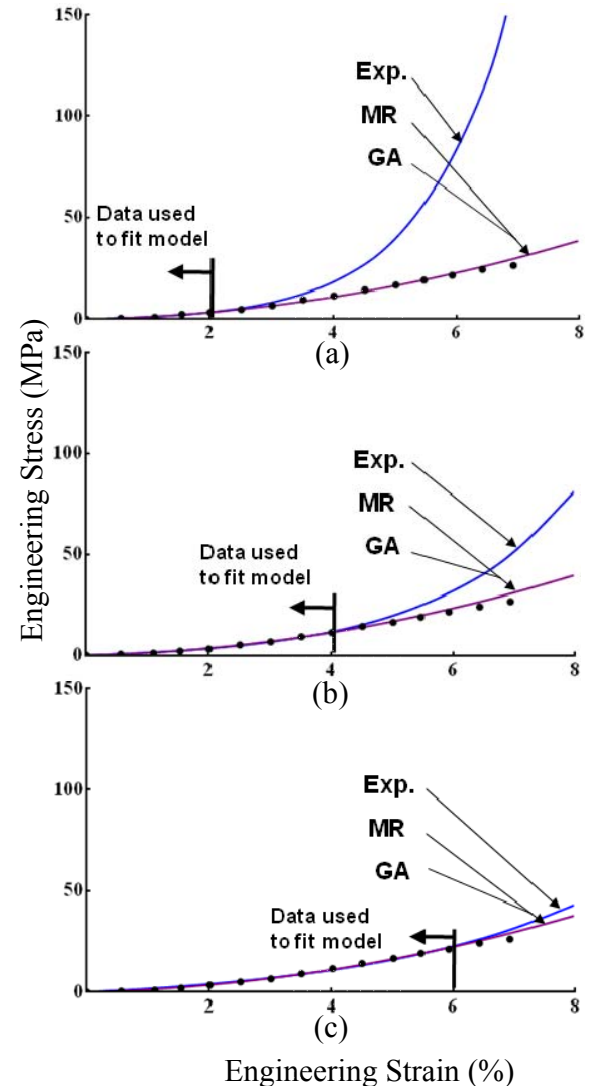

Fig. 1. Engineering stress vs. strain for the $A C L$. The experimental data (Woo et al. 1992) were used to fit the models to three different levels of strain $2 \%$ (a), $4 \%$ (b), and $6 \%$ (c).

When the models are fit to the stress-relaxation data for ACL, all of the models closely match the experimental data. Since the previous studies in this field have been mainly investigated through derivativebased approaches, it should be noted that in the proposed study, the focus on a derivative-free approach is preferable and the investigation of GA as a directsearch tool is desirable. During the approximation process based on the GA, the trend of curve matching can be tracked through generations by determining its behavior based on fitness function value and average distance between individuals as well as the curve matching quality analysis in order to fit the experimental data.

The GA satisfies the requirements of an alternative for minimally invasive measurement to determine the QLV parameters. The convergence ability of this method can be adjusted according to the required precision by determining the fitness function limit as one of the stopping criteria.
For time-saving factor, the two sets of experiments including the convergence rate and curve matching quality are investigated. The first investigation is focused on defining the weight coefficient around the change point of data portion from the ramping phase to the relaxation phase of diagram. Similarly, the second investigation is focused on defining the appropriate slope for the relaxation phase in accordance with the time-dependent reduction of stress at the end of the time period (1600s). As illustrated in Fig. 2, the performance of GA for both cases indicates that these two techniques can get the desired curve matching with fewer generations. However, the latter solution (Fig. 2a) tends to get the optimally estimated QLV parameters with fewer generations in comparison with the former solution (Fig. 2-b). It should also be noted that the estimation of upper and lower bounds for starting the search algorithm is effective to get faster convergence solutions. With the ability provided by these two techniques and adjusting the optimal fitness function limit, the proposed GA provides a systematic gradientfree solution for minimally invasive measurement to determine the QLV parameters during the reasonable time period.

Apart from the performance study of proposed GA method in aspect of curve matching, this estimation method has been investigated through two different crossover functions including single point conventional operator with less than 60 percent fraction value and direction based crossover (DBXO). Since desired fitness function value using GA method with conventional single point crossover operator has been obtained after averagely 50 generations, the direction based crossover function (Eq. 13) has been clearly considered to work more efficiently with regard to an optimal timing up to getting the same accuracy through less than 20 generations.

\section{Discussion}

In this study, the ability of GA is comprehensively evaluated to estimate and predict the QLV parameters of the ACL in order to define a systematic gradientfree bootstrapping alternative for minimally invasive measurement. This evaluation process uses three different constitutive laws to describe the elastic stress response through a gradient-based method and a gradient-free direct-search tool. The approximation performance of GA for the estimation of QLV parameters are elucidated as a practical systematic alternative for minimally invasive measurement. However, great care should be taken when defining the probability of crossover and elitism strategy, as well as the ranking method of selection GA operator.

Since the objective of this study is to primarily evaluate the improved gradient-free approach using GA as a direct-search tool, the comparison of this 
approach with the previously evaluated methods using reliable experimental data is accordingly chosen to model QLV parameters. Otherwise, future 3D nonlinear models may need to apply an inverse solution, i.e. finite element, to be applied for characterization of soft tissue properties from the

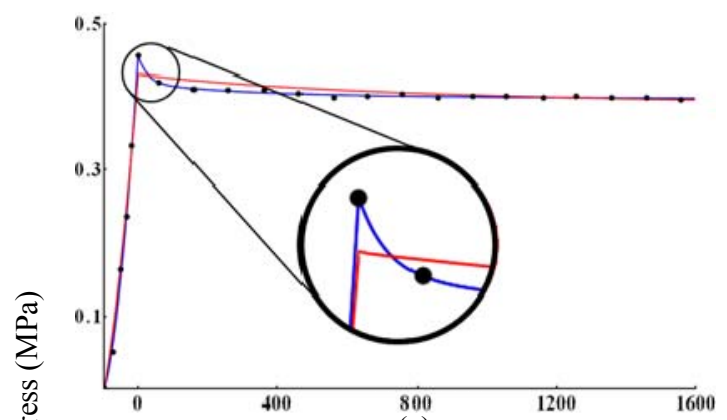

(a)

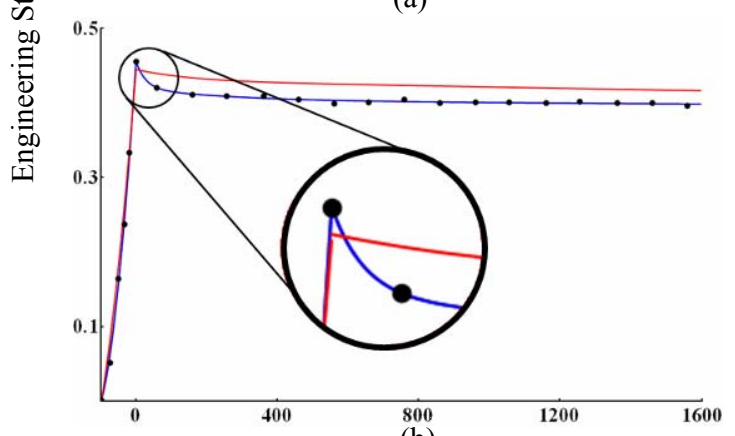

(b)

Time (s)

Fig. 2. Engineering stress vs. time for the $A C L$. The experimental data are depicted by the dots (Pioletti et al. 1998, 2000). The fitted models at $2 \%$ strain with focusing on (a) change point (peak stress point) and (b) relaxation portion are indicated by red lines.

experimental data obtained by in-situ robot-assisted measurements to estimate the stress-strain behavior in response to different loading conditions such as cyclic stress relaxation and creep. Measurement of in-situ stress and strain poses extreme experimental difficulties and this data cannot be obtained directly from the measured displacements and forces. Defining an appropriate methodology and implementation of an applicable robotic indenter are also the challenging tasks in this field.

\section{Acknowledgement}

This work was supported in part by the Australian Research Council (ARC) Discovery Project under grant DP0986814. The authors would like to thank L.E. DeFrate, Ph.D., for his valuable comments regarding his previous studies.

\section{References}

[1] Basdogan C, De S, Kim J, Muniyandi M, Srinivasan MA (2004), Haptic in minimally invasive surgical simulation and training, IEEE Computer Graphics and Appls 24(2): 56-64.

[2] Samur E, Sedef M, Basdogan C, Avtan L, Duzgun O (2007),A robotic indenter for minimally invasive measurement and characterization of soft tissue response, Medical Image Analysis 11: 361-373.

[3] C. Basdogan, M. Sedef, M. Harders, S. Wesarg, VR-based simulators for training in minimally invasive surgery, IEEE Computer Graphics and Applications, 27(2), 2007, pp57-66.

[4] Zhong Y, Shirinzadeh B, J. Smith (2007), Soft Tissue Deformation with Neural Dynamics for Surgery Simulation, Intl J Robotics and Automation 22(1):1-9.

[5] Zhong Y, Shirinzadeh B, Alici G, Smith J (2006), A Neural Network Methodology for Deformable Object Simulation, IEEE Trans IT in Biomedicine 10(4):749-762.

[6] Provenzano P, Lakes R, Keenan T, Vanderby R Jr (2001), Nonlinear ligament viscoelasticity, Ann Biomed 29:908-914.

[7] DeFrate LE, van der Ven A, Boyer PJ, Gill TJ, Li G (2006) The measurement of the variation in the surface strains of Achilles tendon grafts using imaging techniques. J Biomech 39:399-405.

[8] Pioletti DP, Rakotomanana LR, Benvenuti JF, Leyvraz PF (1998), Viscoelastic constitutive law in large deformations: application to human knee ligaments and tendons, J Biomech 31:753-757.

[9] Holzapfel GA (2000), Nonlinear solid mechanics: a continuum approach for engineering, Wiley, Chichester, New York, pp xiv, 455.

[10] Abramowitch SD, Woo SL (2004), An Improved method to analyse the stress relaxation of ligaments following a finite ramp time based on the quasi-linear viscoelastic theory, J Biomech Eng 126: 92-97.

[11] Kohandel M, Sivaloganathan S, Tenti G (2008), Estimation of the quasi-linear viscoelastic parameters using a genetic algorithm, Math and Comp Modeling 47: 266-270.

[12] DeFrate LE, Li G (2007), The prediction of stress-relaxation of ligaments and tendons using the quasi-linear viscoelastic model, Biomechan Model Mechanobiol 6:245-251.

[13] Fung YC (1981), Biomechanics: mechanical properties of living tissues. Springer, Berlin Heidelberg New York, pp xii, 433.

[14] Sinha, Naresh K, Zadeh Lotfi A, Gupta, Madan M (2000), Soft Computing and Intelligent Systems: Theory and Applications Ch.6, ISBN-13: 9780126464900 , Academic Press.

[15] Pioletti DP, Rakotomanana LR (2000), On the independence of time and strain effects in the stress relaxation of ligaments and tendons, J Biomech 33:1729-1732. 